\title{
Hypoperfusive and hypertensive ocular manifestations in Takayasu arteritis
}

This article was published in the following Dove Press journal:

Clinical Ophthalmology

13 October 2010

Number of times this article has been viewed

\author{
Jayanthi Peter' \\ Sarada David' \\ George Joseph ${ }^{2}$ \\ Saban Horo' \\ Debashish Danda ${ }^{3}$ \\ 'Department of Ophthalmology, \\ Schell Eye Hospital, Vellore, India; \\ ${ }^{2}$ Department of Cardiology, Christian \\ Medical College Hospital, Vellore, \\ India; ${ }^{3}$ Department of Clinical \\ Immunology and Rheumatology, \\ Christian Medical College Hospital, \\ Vellore, India
}

\begin{abstract}
Takayasu arteritis is a relatively rare inflammatory arteritis that can be associated with ocular manifestations. We report four patients with proven Takayasu arteritis; two patients manifested hypoperfusive ocular manifestations of ocular ischemic syndrome and anterior ischemic optic neuropathy whilst two others had exudative retinal detachment and papilledema as a result of severe hypertension. The ischemic ocular manifestations were a result of hypoperfusion of the ocular structures due to occlusive arteritis of the aortic arch and its branches. The exudative retinal detachment and papilledema were manifestations of severe hypertension due to renal arterial involvement. Patients with Takayasu arteritis should be referred for ophthalmic assessment and screening for hypoperfusive and hypertensive manifestations.
\end{abstract}

Keywords: Takayasu, retinopathy, ocular ischemic syndrome, India

\section{Introduction}

Takayasu arteritis is an inflammatory arteritis involving the large arteries. ${ }^{1}$ Ocular manifestations are not uncommon in Takayasu arteritis. ${ }^{2}$ Occlusive arteritis of the aortic arch branches results in ischemic ocular manifestations, whilst involvement of the renal artery or supra-renal aorta causes eye manifestations, due to severe and uncontrolled hypertension. The best described ischemic ocular manifestation in Takayasu arteritis is Takayasu retinopathy. Takayasu retinopathy had been classified into four stages by Uyama and Asayma. ${ }^{3}$ Stage 1 Takayasu retinopathy is characterized by distension of veins, stage 2 by micro-aneurysm formation, stage 3 by the formation of arterio-venous anastomoses and stage 4 by the presence of ocular complications like cataract, rubeosis iridis, retinal ischemia, neovascularization and vitreous hemorrhage. Other ischemic manifestations like anterior ischemic optic neuropathy, ${ }^{4,5}$ central retinal artery occlusion ${ }^{6}$ and ocular ischemic syndrome have been described infrequently. ${ }^{7,8}$ We describe two hypoperfusive and two hypertensive manifestations in patients with Takayasu arteritis, diagnosed by the American College of Rheumatology classification. ${ }^{9}$ Each patient had a peripheral angiogram to delineate the type of Takayasu arteritis. ${ }^{10}$ In Type I Takayasu arteritis, the inflammatory process is localized to the arch of the aorta and its branches. In Type II disease the lesions involve both the ascending aorta, aortic arch and its branches (Type II a) or in addition the thoracic descending aorta (Type II b). In Type III disease, the thoracic descending aorta is involved along with the abdominal aorta and/or renal arteries. Abdominal aorta involvement or renal artery involvement is classified as Type IV disease, whilst Type V Takayasu arteritis involves the entire aorta and its branches. ${ }^{10}$

Case 1: A 25-year-old female with Type I Takayasu arteritis, presented with gradual progressive painless visual loss in the left eye of 1-year duration. There was
Correspondence: Jayanthi Peter Schell Eye Hospital, Christian Medical College and Hospital, Arni Road, Vellore $63200 \mathrm{I}$, India

Tel $+9|4| 6228|20|$

E-mail peterjohnvictor@yahoo.com.au 
no history of ocular pain or redness. Her best corrected visual acuity was $6 / 12$ in the right eye and $6 / 60$ in the left eye. Anterior and posterior segments of the right eye were within normal limits. Anterior segment of the left eye showed relative afferent pupillary defect, flare and cells $(1+)$ and iris neovascularisation. Intraocular pressure was $14 \mathrm{mmHg}$ in both eyes. Gonioscopy revealed closed angle anatomy in both eyes. Fundus examination of the left eye revealed disc pallor, arteriolar attenuation without neovascularisation or retinal hemorrhages. Fundus angiogram was normal in the right eye (Figure 1b) except for an increased arterio-venous time. The left eye fundus fluorescein angiogram in the venous phase showed multiple microaneurysms and areas of capillary non-perfusion and arteriolar attenuation consistent with ocular ischemic syndrome (Figure 1a). The reason for the reduced visual acuity in the right eye could not be readily explained. The increased arterio-venous time could represent very early ocular ischemic syndrome in the right eye also, albeit no other changes were evident on the fundus examination and in the angiogram. The normal intraocular pressure despite closed angles could represent hypoperfusion of the ciliary body and reduced aqueous secretion. Pan-retinal photocoagulation was performed for the left eye to prevent neovascular glaucoma.

Case 2: A 29 year-old female presented with a history of painless visual loss in the left eye of 3 years' duration. She had right-sided hemiparesis, secondary to a cerebrovascular accident involving the left middle cerebral artery territory, just before she started developing decreased vision. She was diagnosed to have Type I Takayasu arteritis. Her best corrected visual acuity was 6/6 in the right eye and hand movements in the left eye. Anterior segment was normal in the right eye while the left eye revealed 15 degree exotropia, relative afferent pupillary defect and an early posterior subcapsular cataract. Intraocular pressures were $14 \mathrm{mmHg}$ in both eyes. Gonioscopy revealed open angles. A dilated fundus examination showed hypertensive retinopathy in the right eye and optic atrophy in the left eye. Humphrey's field analyzer showed right homonymous hemianopia secondary to the cerebrovascular accident that the patient had a few years ago. Fundus fluorescein angiogram showed normal perfusion to the right eye and disc hypo-fluorescence due to loss of capillary perfusion in the left eye. The venous phase of the fundus angiogram of the left eye is depicted in Figure 2. A diagnosis of optic atrophy probably secondary to anterior ischemic optic neuropathy was made. Aortic angiogram revealed stenosis of both subclavian and carotid arteries. Although the right subclavian artery was involved, circulation to the right eye was not compromised. However the left eye circulation was compromised sufficiently to cause an anterior ischemic optic neuropathy. The angioplasty and stenting of the subclavian and carotid arteries relieved the obstruction in the subclavian arteries and preserved circulation in the right eye even after 3 years following revascularization. The left eye visual acuity, as expected, remained unchanged despite angioplasty and stenting of the left subclavian and carotid arteries.

Case 3: A 13 year-old girl presented with dizziness and seizures for 3 months and decreased vision of both eyes of 1-month duration. An aortic angiogram was suggestive of Type III Takayasu arteritis. Her best corrected visual acuity was 2/60 in the right eye and 5/60 in the left eye. Intraocular pressure was $14 \mathrm{mmHg}$ in both eyes. Dilated fundus examination revealed bilateral exudative retinal detachment (Figure 3) involving the macula. Systemic
A

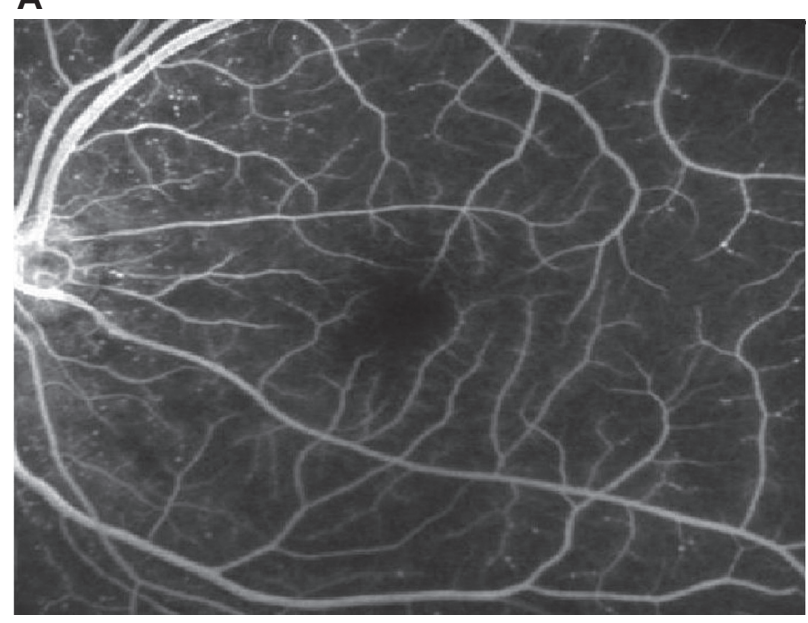

B

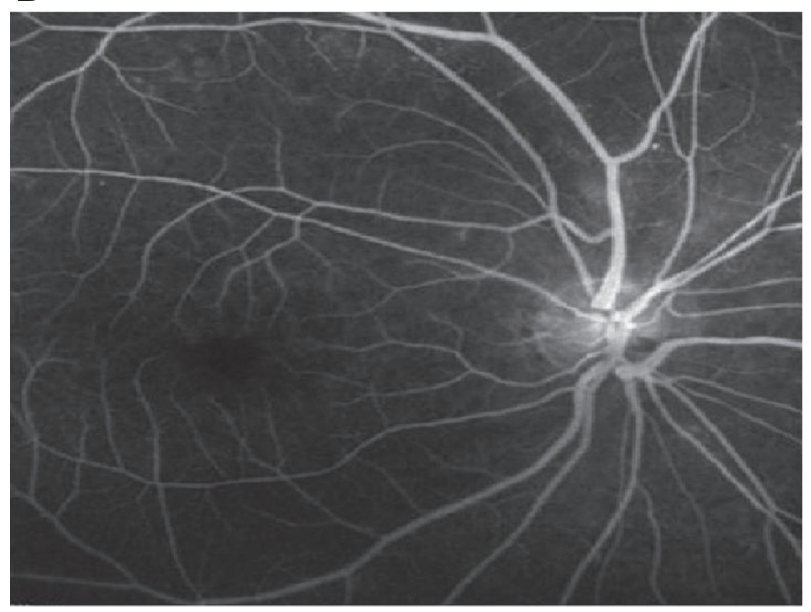

Figure I A) Fundus angiogram in the venous phase demonstrating multiple microaneurysms, areas of capillary non-perfusion and arteriolar attenuation in the left eye. B) Normal fundus angiogram of the right eye. 


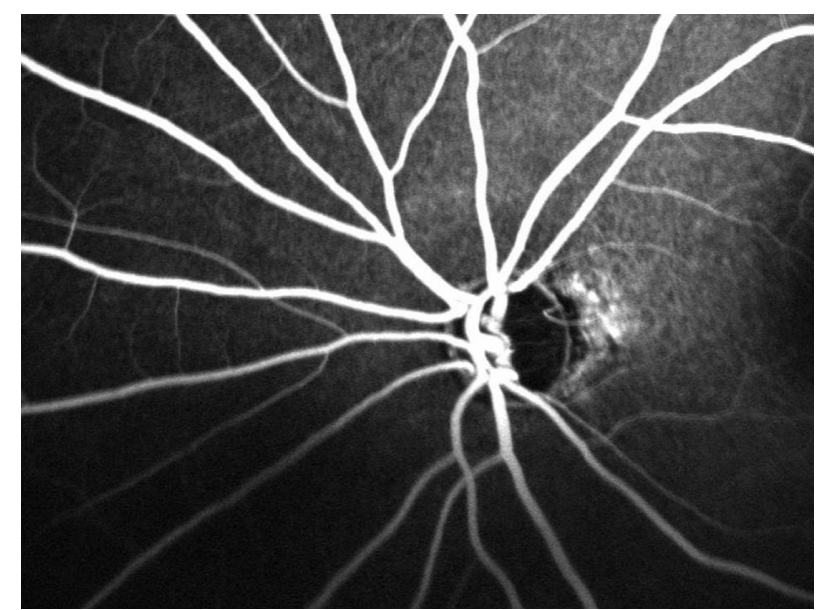

Figure 2 Fundus angiogram of the left eye. Venous phase of the fundus angiogram demonstrating disc hypo-fluorescence due to loss of capillary perfusion in the left eye.

examination showed systolic blood pressure difference of $20 \mathrm{~mm}$ between the upper limbs $(170 / 100 \mathrm{mmHg}$ [right] and 150/100 mmHg [left]). She was started on anti-hypertensive drugs and immunosuppressants. She subsequently underwent angioplasty with stenting of both renal arteries and infra-renal aorta as well as angioplasty of both subclavian arteries. Three months later, her blood pressure was under control and the best corrected visual acuity was $6 / 18$ in both the eyes with total resolution of the retinal detachment.

Case 4: A 28 year-old male, diagnosed with Type V Takayasu arteritis, presented with gradual decrease in vision in both eyes for 2 months. His blood pressure was $200 / 120 \mathrm{mmHg}$. His vision was $6 / 24$ in both eyes. The anterior segments were normal. A dilated fundus examination revealed bilateral papilledema (Figure 4). A magnetic resonance imaging (MRI) scan at that time did not reveal

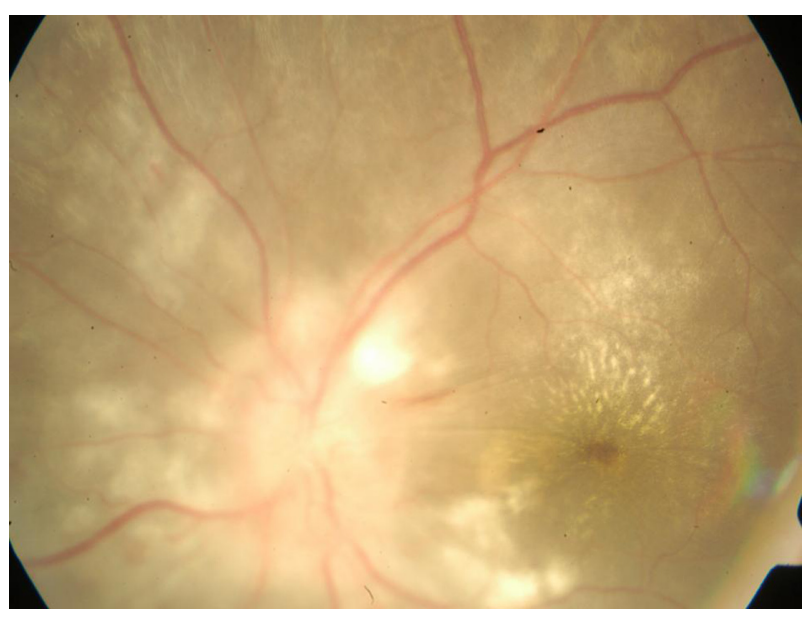

Figure 3 Fundus photograph of the left eye showing exudative retinal detachment and macular star.

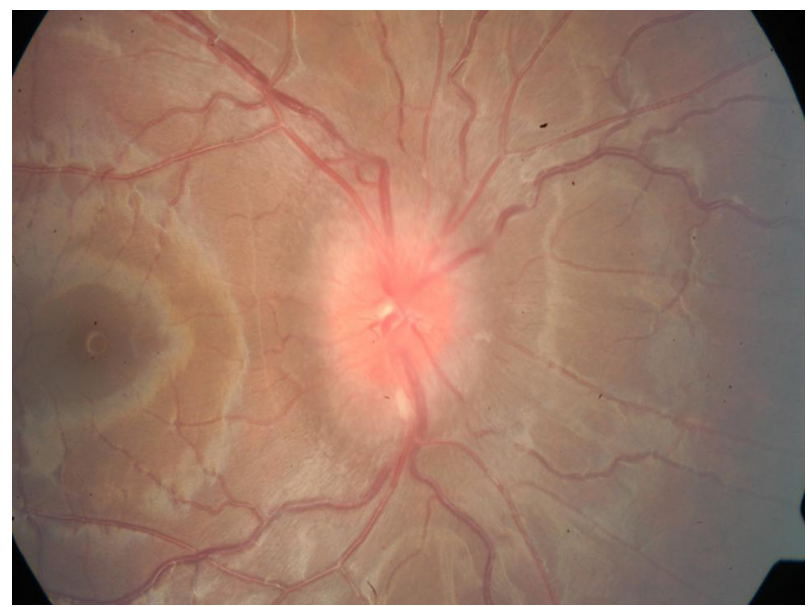

Figure 4 Fundus photograph of the right eye showing papilledema.

any intracranial pathology to account for the papilledema. A peripheral angiogram revealed bilateral renal artery stenosis and abdominal aortic aneurysm. His blood pressure control, effected by anti-hypertensive drugs and renal artery stenting, led to resolution of papilledema and maintenance of visual acuity.

\section{Discussion}

Takayasu's arteritis is an inflammatory vascular disease of the young and involves primarily the large arteries. Classically Takayasu arteritis involves the aorta and its major branches, namely subclavian, renal or carotid arteries and is occasionally called the "pulseless disease," as there is difficulty in detecting peripheral pulses due to vascular narrowing. ${ }^{1}$ The disease affects more females than males and usually begins in the second or third decade of life. The estimated incidence of Takayasu arteritis is 2.6 cases per million persons per year, and most new patients are women of reproductive age, with an Asian or Hispanic ethnic background. ${ }^{4}$

There have been very few reports of ocular ischemic syndrome $e^{7,8}$ and anterior ischemic optic neuropathy ${ }^{4,5}$ occurring in association with Takayasu arteritis. These four unique ocular manifestations of Takayasu arteritis were seen in a single center over a 1-year period. This may be attributed to the fact that our institution is a referral center for ophthalmology, interventional cardiology and rheumatology.

Takayasu arteritis occurs due to an autoimmune process that results in a chronic granulomatous inflammation. ${ }^{1}$ This causes attenuation and obliteration of the branches of the aorta with or without thrombosis leading to cerebral and ocular ischemia. ${ }^{2}$ The ischemic retinal changes in Takayasu arteritis depend on which portions of the carotid arteries become occluded, the rate of development and duration 
of ocular vascular insufficiency, and the effectiveness of collateral blood supply to the eye. ${ }^{2}$ The asymmetry of ocular manifestations in Cases 1 and 2, despite bilateral involvement of the major branches of the aorta, is thus not surprising. Occlusion of the branches of the aortic arch resulted in anterior ischemic optic neuropathy and ocular ischemic syndrome in the patients described in this report.

Stenosis of the descending aorta and its branches can cause severe hypertension due to renal artery involvement. Two of our patients manifested consequences of severe hypertension related to renal artery involvement in Takayasu arteritis, proven by angiography. The presence of bilateral papilledema without other changes of hypertensive retinopathy in Case 4 was unusual. However no other intracranial cause of papilledema was evident on a MRI scan of the brain. Bilateral optic disc swelling has been reported in a child with severe hypertension due to pheochromocytoma without any other evidence of Grade 4 hypertensive retinopathy. ${ }^{11}$ In the absence of other causes of papilledema and the resolution of the papilledema with control of hypertension, the papilledema was attributed to severe hypertension.

These cases highlight the fact that patients with Takayasu arteritis can present with either hypoperfusive ocular manifestations or hypertensive ocular manifestations and that these manifestations could lead to significant visual loss. Given that a majority of these patients are young (all patients in our series were less than 30 years of age), the consequence of a sight threatening ocular complication/manifestation of Takayasu arteritis can be devastating. Whilst some of these ocular manifestations may be acute in onset, like the acute visual loss in our patient with anterior ischemic optic neuropathy, others can be sub-acute as in the patient who had hypertensive retinopathy due to uncontrolled hypertension as a result of renal artery involvement by the arteritis. In other patients, the ocular symptoms may be more gradual and chronic, paralleling the gradual ischemia that develops due to progressive vascular occlusion of the arteries as evidenced in our patient with ocular ischemic syndrome. Whilst acute sight loss, like in anterior ischemic optic neuropathy, may not be amenable to therapy that could restore sight (despite restoration of blood flow), other sub-acute or chronic ocular manifestations are eminently treatable. Ophthalmic intervention for ocular neovascularization can be done in the form of laser therapy. Medical treatment in the form of disease modifying drugs such as steroids and immunosuppressants alter the course of the disease whilst adequate anti-hypertensive therapy prevents the consequences of severe hypertension. Circulation to ischemic areas can be either restored or improved by angioplasty and stenting of occluded arteries.

\section{Disclosure}

The authors report no conflicts of interest in this work.

\section{References}

1. Panja M, Mondal PC. Current status of aortoarteritis in India. J Assoc Physicians India. 2004;52:48-52.

2. Chun YS, Park S, Park I, Chung H, Lee J. The clinical and ocular manifestations of Takayasu arteritis. Retina. 2001;21:132-140.

3. Uyama M, Asayma K. Retinal vascular changes in Takayasu's disease (Pulseless disease). Occurrence and evolution of the lesion. Doc Ophthalmol Proc Series. 9:549-554.

4. Schmidt MH, Fox AJ, Nicolle DA. Bilateral anterior ischemic optic neuropathy as a presentation of Takayasu's disease. J Neuroophthalmol. 1997;17:156-161.

5. Malik KP, Kapoor K, Mehta A, et al. Bilateral anterior ischaemic optic neuropathy in Takayasu arteritis. Indian J Ophthalmol. 2002;50:52-54.

6. Kaushik S, Gupta A, Gupta V, Jain S, Lal V. Retinal arterial occlusion in Takayasu's arteritis. Indian J Ophthalmol. 2005;53:194-196.

7. Koz OG, Astes A, Numan Alp M, Gultan E, Karaaslan Y, Kural G. Bilateral ocular ischemic syndrome as an initial manifestation of Takayasu's arteritis associated with carotid steal syndrome. Rheumatol Int. 2007;27:299-302.

8. Worrall M, Atebara N, Meredith T, Mann ES. Bilateral ocular ischemic syndrome in Takayasu disease. Retina. 2001;21:75-76.

9. Arend WP, Michel BA, Bloch DA, et al. The American College of Rheumatology 1990 criteria for the classification of Takayasu arteritis. Arthritis Rheum. 1990;33:1129-1134.

10. Nastri MV, Baptista LP, Baroni RH, et al. Gadolinium-enhanced threedimensional MR angiography of Takayasu arteritis. Radiographics. 2004;24:773-786.

11. Ba-Abbad RA, Nowilaty SR. Bilateral optic disc swelling as the presenting sign of pheochromocytoma in a child. Medscape J Med. 2008;10:176.
Clinical Ophthalmology

\section{Publish your work in this journal}

Clinical Ophthalmology is an international, peer-reviewed journal covering all subspecialties within ophthalmology. Key topics include: Optometry; Visual science; Pharmacology and drug therapy in eye diseases; Basic Sciences; Primary and Secondary eye care; Patient Safety and Quality of Care Improvements. This journal is indexed on Submit your manuscript here: http://www.dovepress.com/clinical-ophthalmology-journal

\section{Dovepress}

PubMed Central and CAS, and is the official journal of The Society of Clinical Ophthalmology (SCO). The manuscript management system is completely online and includes a very quick and fair peer-review system, which is all easy to use. Visit http://www.dovepress.com/ testimonials.php to read real quotes from published authors. 